\title{
INRADIUS AND INTEGRAL MEANS FOR GREEN'S FUNCTIONS AND CONFORMAL MAPPINGS
}

\author{
RODRIGO BAÑUELOS, TOM CARROLL, AND ELIZABETH HOUSWORTH
}

(Communicated by Albert Baernstein II)

\begin{abstract}
Let $D$ be a convex planar domain of finite inradius $R_{D}$. Fix the point $0 \in D$ and suppose the disk centered at 0 and radius $R_{D}$ is contained in $D$. Under these assumptions we prove that the symmetric decreasing rearrangement in $\theta$ of the Green's function $G_{D}\left(0, \rho e^{i \theta}\right)$, for fixed $\rho$, is dominated by the corresponding quantity for the strip of width $2 R_{D}$. From this, sharp integral mean inequalities for the Green's function and the conformal map from the disk to the domain follow. The proof is geometric, relying on comparison estimates for the hyperbolic metric of $D$ with that of the strip and a careful analysis of geodesics.
\end{abstract}

\section{INTRODUCTION}

Let $D$ be a simply connected domain in the complex plane. Let $R_{D}$ be the supremum of the radii of all disks contained in $D$. This geometric quantity is called the inradius of the domain $D$. Many interesting and challenging extremal problems arise when one fixes $R_{D}$ and asks for those domains which maximize or minimize various quantities which depend on $D$. For example, one such problem which has been extensively studied and which remains open is the following: amongst all simply connected domains with inradius 1, find the one(s) which has the lowest fundamental frequency. It is shown in [2] that this problem is closely related to problems concerning the maximum expected lifetime of Brownian motion in the domain, the schlicht Bloch-Landau constant, and integral means for the derivative of the conformal mapping from the disk to the domain.

Some of these problems can be in turn stated as problems for integrals and integral means of the Green's function for $D$. More precisely, let $G_{D}(w, z)$ be the Green's function for $D$ and let $\varphi$ be a nonnegative, nondecreasing function on $[0, \infty)$ satisfying $\int_{0}^{\infty} r \varphi(\log (\operatorname{coth} r)) d r<\infty\left(\operatorname{such}\right.$ as $\varphi(x)=x^{p}$ for any $\left.p>0\right)$. We denote Lebesgue area measure by $m$. The argument in [2], p. 583 (equation (1.7)), will

Received by the editors August 23, 1996.

1991 Mathematics Subject Classification. Primary 30C45.

Key words and phrases. Integral means, Green's functions, symmetric decreasing rearrangements, Baernstein star functions.

Research of the first author supported in part by NSF under grant DMS9400854, of the second author by the President's Research Fund, University College, Cork, and of the third author by NSF under grant DMS9501611. 
give that for such $\varphi$ 's,

$$
\sup _{w \in D} \int_{D} \varphi\left(G_{D}(w, z)\right) d m(z)<\infty \quad \text { if and only if } \quad R_{D}<\infty .
$$

Taking account of (1) and of the classical isoperimetric-type inequality which states that for any domain $D$ and any nondecreasing function $\varphi$,

$$
\sup _{w \in D} \int_{D} \varphi\left(G_{D}(w, z)\right) d m(z) \leq \int_{D^{*}} \varphi\left(G_{D^{*}}(0, z)\right) d m(z),
$$

where $D^{*}$ is the disk centered at the origin with the same area as $D([1]$, p. 61$)$, it is natural to inquire about the following

Extremal Problem: Amongst all simply connected domains $D$ with inradius 1, find those which maximize the integral in (1).

For arbitrary simply connected domains this problem (as well as the other problems mentioned above) are open even for $\varphi(x)=x$, which is the case of the lifetime of Brownian motion in $D$. We refer the reader to [2] for more on this and related problems.

If we restrict our attention to convex domains, then the strip of the given inradius $R_{D}$ is known to be extremal for several of the problems mentioned above. In particular, Sperb [9] showed that for convex domains $D$ with finite inradius,

$$
\sup _{w \in D} \int_{D} G_{D}(w, z) d m(z) \leq \int_{S} G_{S}(0, z) d m(z),
$$

where $S$ is an infinite strip of the same inradius as $D$ and center 0 . Sperb's proof of (3) is based on a maximum principle applied to an appropriately constructed " $P$-function" for the solution of the torsion problem

$$
\begin{cases}\triangle u=-2 & \text { in } D \\ u=0 & \text { on } \partial D .\end{cases}
$$

The proof does not generalize to other $\varphi$ 's. In [3], Bañuelos and Housworth proved that for any convex $D$ with finite inradius $R_{D}$, and any nondecreasing $\varphi$,

$$
\sup _{w \in D} \int_{D} \varphi\left(G_{D}(w, z)\right) d m(z) \leq\left(\frac{2 \pi^{2}}{(\Gamma(1 / 3))^{3}}\right)^{2} \int_{S} \varphi\left(G_{S}(0, z)\right) d m(z)
$$

where $S$ is a strip with center 0 and inradius $R_{D}$. The constant $\left(2 \pi^{2} /(\Gamma(1 / 3))^{3}\right)^{2}$ is approximately 1.0541 . We suspect that this constant should be 1 . In this paper, we consider the integral on the left-hand side of (4) at the point $w$ which is the center of the largest disk in $D$ when such a disk exists. One expects the supremum in (4) to occur at this point although we have been unable to prove this. We comment more on the difficulty of this problem at the end of the paper. What we prove here is that at this point $w$, the integral on the left-hand side of (4) is bounded by the integral on the right-hand side where the constant is replaced by 1 . This is a direct consequence of Corollary 1 below. In fact, we prove considerably stronger results concerning the symmetric decreasing rearrangements and integral means for the Green's function. Our arguments here are completely different from those in [3].

Assumptions: Throughout this paper our assumptions will be, unless specifically stated otherwise, that $D$ is a convex planar domain with inradius $R_{D}$ and that $D$ contains a largest disk centered at $0 \in D$. When making comparisons between 
functions involving $D$ and functions involving a strip $S$, we always assume that the strip has the same inradius as $D$ and is centered at 0 .

The center of the largest disk in $D$ is also called the incenter of $D$. All results hold trivially for convex domains of finite inradius that do not contain a largest disk because such domains are contained in a strip of the same inradius.

Recall that the distribution function of a real-valued function $g$ integrable over $[-\pi, \pi]$ is given by $\lambda(t)=|\{x: g(x)>t\}|$ and that the symmetric decreasing rearrangement of $g$ is given by $\tilde{g}(x)=\min \{t: \lambda(t) \leq 2 x\}$ for $0 \leq x \leq \pi$ with $\tilde{g}(-x)=\tilde{g}(x)$. As is standard, for $z$ in $D$, we set $G_{D}(z, w)=0$ when $w$ lies in the complement of $D$.

Theorem 1. Let $\tilde{G}_{D}\left(0, \rho e^{i \theta}\right)$ be the symmetric decreasing rearrangement in $\theta$ of $G_{D}\left(0, \rho e^{i \theta}\right)$ for fixed $\rho$. Then $\tilde{G}_{D}\left(0, \rho e^{i \theta}\right) \leq \tilde{G}_{S}\left(0, \rho e^{i \theta}\right)$ where $S$ is an infinite strip with center 0 and the same inradius as $D$. If equality holds at any point for which $\tilde{G}_{S}\left(0, \rho e^{i \theta}\right)>0$, then $D$ is an infinite strip.

As a consequence of this theorem, we obtain the following results. The first is straightforward:

Corollary 1. If $\varphi$ is any nonnegative, nondecreasing function on $[0, \infty)$, then $\int_{0}^{2 \pi} \varphi\left(G_{D}\left(0, \rho e^{i \theta}\right)\right) d \theta \leq \int_{0}^{2 \pi} \varphi\left(G_{S}\left(0, \rho e^{i \theta}\right)\right) d \theta$, for any $0<\rho<\infty$. If equality holds for some strictly increasing $\varphi$ and some $\rho$ then $D$ is an infinite strip.

The next corollary, as explained in more detail at the end of the paper, follows from Corollary 1 and the connections between Baernstein star functions and integral means. First, for any real-valued function $g(t)$ on $[-\pi, \pi]$, the Baernstein star function is defined, for $0 \leq \theta \leq \pi$, as

$$
g^{*}(\theta)=\sup _{|E|=2 \theta} \int_{E} g(t) d t \quad \text { where } E \subseteq[-\pi, \pi] .
$$

Note that $g^{*}(\theta)=\int_{-\theta}^{\theta} \tilde{g}(x) d x$.

Corollary 2. Let $f$ be a conformal map of the unit disk onto $D$ which maps 0 to the incenter of $D$ which we assume to be at 0 . Let $\psi$ be a conformal map of the unit disk onto the strip $S$ for which $\psi(0)=0$. Then $(\log |f|)^{*}\left(r e^{i \theta}\right) \leq(\log |\psi|)^{*}\left(r e^{i \theta}\right)$. In particular, for any convex, nondecreasing function $\Phi$ on $(-\infty, \infty)$,

$$
\int_{0}^{2 \pi} \Phi\left(\log \left|f\left(r e^{i \theta}\right)\right|\right) d \theta \leq \int_{0}^{2 \pi} \Phi\left(\log \left|\psi\left(r e^{i \theta}\right)\right|\right) d \theta,
$$

for any $0<r<1$.

\section{Proofs}

In the proof of Theorem 1, we will exploit the connection between the Green's function for a simply connected planar domain $D$ and its hyperbolic metric. If we denote by $U$ the unit disk, then the density of the hyperbolic metric in $D$ is given by $\sigma(z ; D)=1 /\left|F^{\prime}(0)\right|$ where $F$ is a conformal mapping of $U$ onto $D$ with $F(0)=z$. The hyperbolic distance between points $w$ and $z$ in $D$ is defined by

$$
d(w, z ; D)=\inf _{C}\left\{\int_{0}^{1} \sigma(C(t) ; D)\left|C^{\prime}(t)\right| d t\right\}
$$


where the infimum is taken over all rectifiable curves in $D$ with $C(0)=w$ and $C(1)=z$. The minimizing curve $\Gamma$ exists and is called the hyperbolic geodesic between $w$ and $z$. The hyperbolic distance is a conformal invariant in the sense that if $f$ is any conformal map of $U$ onto $D$ then

$$
d(w, z ; D)=d\left(f^{-1}(w), f^{-1}(z) ; U\right) .
$$

For the unit disk, we have that

$$
d(0, z ; U)=(1 / 2) \log [(1+|z|) /(1-|z|)] .
$$

On the other hand, the Green's function for the unit disk with pole at 0 is given by $G_{U}(0, z)=(1 / \pi) \log (1 /|z|)$. Solving for $|z|$, we see that the Green's function and the hyperbolic distance for $U$ are related by

$$
G_{U}(0, z)=\frac{1}{\pi} \log \frac{1+e^{-2 d(0, z ; U)}}{1-e^{-2 d(0, z ; U)}}=\frac{1}{\pi} \log (\operatorname{coth} d(0, z ; U)) .
$$

Since the Green's function is also a conformal invariant, we obtain from (6) and (7) the well-known relationship that for any simply connected planar domain,

$$
G_{D}(w, z)=\frac{1}{\pi} \log (\operatorname{coth} d(w, z ; D)) .
$$

The following result due to Minda allows us to compare hyperbolic distances in our convex domains to hyperbolic distances in the strip via the Euclidean distance to the boundary. By (8), we can then compare the Green's functions.

Theorem A (Minda, [6]). Let $D$ be a convex domain of finite inradius $R_{D}$. Let $\delta_{D}(z)$ be the Euclidean distance from $z$ to the boundary of $D$. Then

$$
\sigma(z ; D) \geq \frac{\pi}{4 R_{D} \sin \left(\pi \delta_{D}(z) /\left(2 R_{D}\right)\right)} .
$$

Furthermore, if equality occurs in (9) at any point $z$ in $D$, then equality occurs for all $z$ and $D$ is a strip of inradius $R_{D}$.

Next we note that any convex domain $D$ containing a largest disk centered at 0 can either be embedded in a strip centered at 0 with the same inradius as $D$ or in a triangle with center 0 and the same inradius as $D$. By subordination, it is therefore enough to prove Theorem 1 for triangles $T$ with vertices $A, B, C$ and corresponding angles $\alpha, \beta, \gamma$ as in Figure 1 .

To prove Theorem 1, we will use the following lemma which may be viewed as a partial counterpart of Theorem A for the hyperbolic metric rather than for its density.

Lemma 1. Suppose that $T$ is a triangle and that $\Gamma$ is the hyperbolic geodesic with respect to $T$ which joins 0 , the center of $T$, to the point $z$ in $T$. Let $S$ be the (not necessarily unique) strip with center 0 which is tangent to $T$ along the side of $T$ nearest $z$. Then each point $w$ on $\Gamma$ is closer, in the Euclidean sense, to the boundary of the triangle $T$ than it is to the boundary of the strip $S$; that is, $\delta_{T}(w) \leq \delta_{S}(w)$.

This implies that $d(0, z ; T) \geq d(0, z ; S)$ and that $G_{T}(0, z) \leq G_{S}(0, z)$. Furthermore, if $d(0, z ; T)=d(0, z ; S)$ at any point $z$ in $T$, then $T$ is a strip of inradius $R_{T}$.

A rearrangement argument will be applied to estimate the contribution from each of the three natural comparison strips in terms of a single strip using the following lemma. 


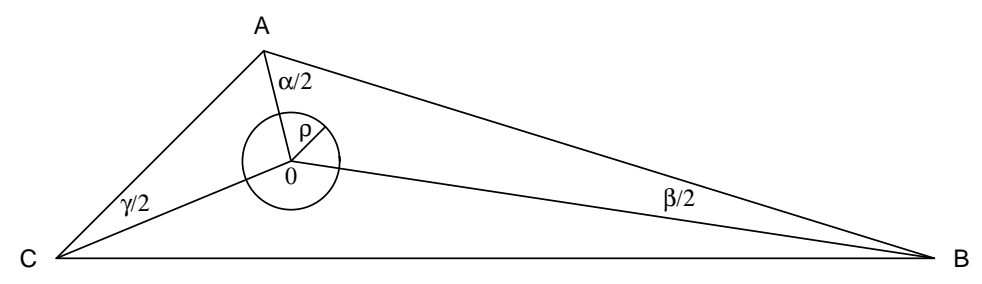

Figure 1. Triangle

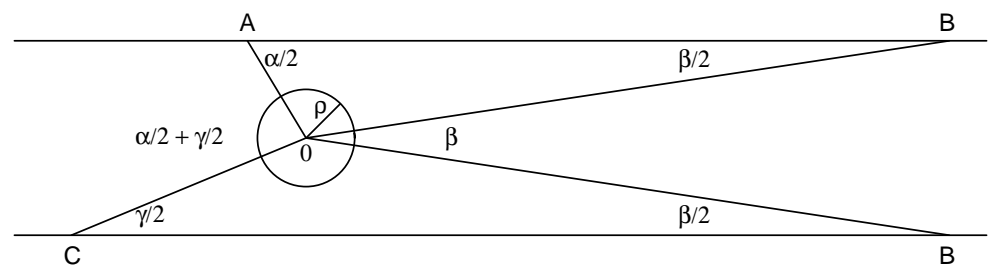

FiguRE 2. Strip

Lemma 2. Let $G_{S}(0, z)$ be the Green's function for a horizontal strip $S$ with pole at 0 , the center of the strip. Then, for each positive $\rho, G_{S}\left(0, \rho e^{i \theta}\right)$ decreases as $\theta$ increases from 0 to $\pi / 2$.

We first show how these two lemmas may be used to obtain Theorem 1. Please refer to Figures 1 and 2.

We rotate both the triangle and the strip so that a side lies along the line $\operatorname{Im}(w)=-R_{T}$. We also assume that the longest side of $T$ is along this line so that $0 A$ is the shortest angle bisector. Lemma 1, the symmetry of the Green's function for the strip, and a cut and paste procedure clearly show that $G_{T}(0, z)$ with $|z|=\rho$ and $z$ lying between the angle bisectors $0 A$ and $0 B$ or between the bisectors $0 B$ and $0 C$ can be dominated by $G_{S}(0, w)$ at a point $w$ in $S$ with $w=\rho e^{i \eta}$ and $\beta / 2<\eta<\pi-\alpha / 2$ or $-\pi+\gamma / 2<\eta<-\beta / 2$, respectively.

Note that if $\rho$ is actually larger than the length of the shortest angle bisector, $0 \mathrm{~A}$, then we have not in fact used the portion of $\{|w|=\rho\}$ in $S$ with $\pi / 2<\eta<\pi+\gamma / 2$. By placing $\triangle A 0 C$ with side $A C$ along the upper boundary of the strip, we can dominate the Green's function along the part of $\{|z|=\rho\}$ in $T \cap \Delta A 0 C$ by that for $S$ along $\{|w|=\rho\}$ with $\pi / 2<\eta<\pi-\gamma / 2$. Thus we are done.

If $\rho$ is less than the length of the shortest angle bisector, then the comparison is made between the points of $\{|z|=\rho\}$ in $T$ between $0 C$ and $0 A$ and another strip $S^{\prime}$, a duplicate of $S$, at the points $w^{\prime}=\rho e^{i \eta^{\prime}}$ where $\alpha / 2<\eta^{\prime}<\pi-\gamma / 2$. We can then see that the Green's function for $S^{\prime}$ at these points can be compared to the Green's function for $S$ in the unused part of $\{|w|=\rho\}$ in $S$ because the unused angle of $\{|w|=\rho\}$ in $S$ is $\beta+\alpha / 2+\gamma / 2=\pi-\alpha / 2-\gamma / 2$ which is exactly what 


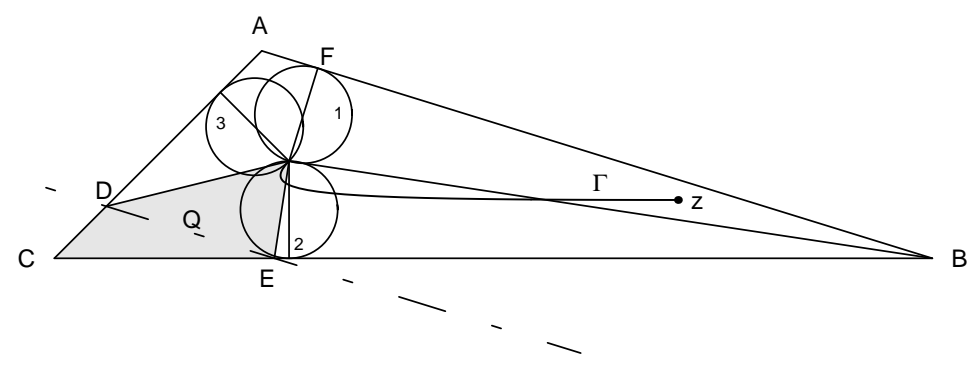

Figure 3. Comparison between distances to the boundaries of $T$ and of $S$

we need. Also, we can make the rearrangement so as to guarantee that the Green's functions increase using Lemma 2. To see that, note that we can compare the Green's function at $w^{\prime}$ in $S^{\prime}$ for $\alpha / 2<\eta^{\prime}<\alpha$ with the Green's function at $w$ in $S$ with $\pi-\alpha / 2<\eta<\pi$. Similarly, the Green's function at $w^{\prime}$ in $S^{\prime}$ for $\alpha<\eta^{\prime}<\alpha+\beta$ can be compared to that for $S$ with $-\beta / 2<\eta<\beta / 2$. Finally, the Green's function at $w^{\prime}$ in $S^{\prime}$ for $\pi-\gamma=\alpha+\beta<\eta^{\prime}<\pi-\gamma / 2$ can be compared to that for $S$ with $\pi<\eta<\pi+\gamma / 2$.

Since, by Lemma 2, the Green's function only increases with each of these rearrangements we see that, on each of these arcs, the Green's function for the triangle is dominated by that for the strip on an equivalent arc. Thus, for each $t$,

$$
\left|\left\{\eta: G_{T}\left(0, \rho e^{i \eta}\right)>t\right\}\right| \leq\left|\left\{\eta: G_{S}\left(0, \rho e^{i \eta}\right)>t\right\}\right|
$$

and it follows from the definition that the symmetric decreasing rearrangement of $G_{T}\left(0, \rho e^{i \theta}\right)$ is dominated by that of the strip.

To complete the proof of Theorem 1, note that what we have shown above is that for all triangles $T$ and all points $z=\rho e^{i \theta}$ in $T$,

$$
G_{T}\left(0, \rho e^{i \theta}\right)<G_{S}\left(0, \rho e^{i h(\theta)}\right)
$$

for some piecewise linear function $h$, which depends on $\rho$, with strict inequality by Lemma 1 whenever $T$ is really a triangle and not a strip. Thus $\tilde{G}_{T}\left(0, \rho e^{i \theta}\right) \leq$ $\tilde{G}_{S}\left(0, \rho e^{i \theta}\right)$ with the strict inequality whenever $\tilde{G}_{T}\left(0, \rho e^{i \theta}\right) \neq 0$. This extends trivially to hold whenever $\tilde{G}_{S}\left(0, \rho e^{i \theta}\right) \neq 0$. As noted before, this proves Theorem 1 for all convex domains $D$ of finite inradius by subordination since Green's functions increase as the domains increase.

All that remains is to prove the lemmas which we will now do.

Proof of Lemma 1. We refer the reader to Figure 3.

We must show that the hyperbolic geodesic $\Gamma$ from 0 to $z$ in $T$ is always closer, in the Euclidean sense, to the boundary of $T$ than it is to the boundary of the strip with center 0 and the same inradius whose side lies along the side of $T$ closest to $z$. Assume that $z \in \triangle A 0 B$ and that the side of the comparison strip not tangent to $T$ crosses $T$ at points $D$ and $E$. First note that $0 E \perp 0 B$ and that $0 E$ bisects the angle $\angle D E B$. This is an elementary trigonometric argument that can be seen by extending $0 E$ through side $A B$ and using triangle similarities to determine that $\angle 0 E B=(\pi-\beta) / 2=\angle 0 E D$. Since $0 E$ bisects $\angle D E B$, for points along $0 E$, the distance to the boundary of the strip is equal to the distance to the boundary of 
the triangle. Similarly for points along $0 D$. Thus, we must show that the geodesic does not enter the quadrilateral $Q=0 D C E$.

For each side of the triangle, we consider the perpendicular line segment from 0 to that side and construct a disk whose center is the midpoint of the perpendicular and which has radius $R_{T} / 2$. The intersection of these three disks is 0 where the geodesic begins. We can assume by the symmetry in our arguments that $z$ lies in $\triangle F 0 B$ where $F$ is the point at which the perpendicular from 0 crosses side $A B$.

It is known (see [8], Section 4.6, Exercise 1) that the intersection of a geodesic and a closed disk is connected. Thus the geodesic can enter at most two of these disks after leaving the origin. Also note that once it exits one of these disks, it cannot enter it again.

If $z$ is in disk 1 , then the geodesic cannot leave that disk and thus does not enter $Q$. If $z$ is not in disk 1 but the geodesic exits the union of the three disks through disk 1 , then it also never entered $Q$. The only other possibility is that the geodesic exits the union of the three disks through disk 2 . In this case, if it enters the problem quadrilateral $Q$, then it exits through side $0 E$.

Because the geodesic is smooth, starts at 0 , and crosses side $0 E$, at some point, $w$, along the geodesic, the tangent to the geodesic at $w$ is parallel to side $0 E$. At this point, we draw the largest possible disk tangent to the geodesic that will fit inside $T$ and contains part of $0 E$.

Since $0 E \perp 0 B$, this tangent disk will touch side $B C$ of the triangle. This is because the center of the disk is on a line through $w$ and perpendicular to $0 E$ and thus parallel to $0 B$. Consequently, the center of the disk is in $\triangle B 0 C$ and the disk must touch side $B C$. This contradicts Proposition 4.22 in [8] which says that if an open disk is tangent to the hyperbolic geodesic and lies inside the domain, then the geodesic does not intersect the interior of the disk. Since this tangent disk cuts the geodesic off from $z$, we obtain a contradiction.

To see how this implies $G_{T}(0, z) \leq G_{S}(0, z)$, recall that Minda's inequality (9) implies that for $w$ on $\Gamma$,

$$
\sigma(w ; T) \geq \frac{\pi}{4 R_{T} \sin \left[\pi \delta_{T}(w) /\left(2 R_{T}\right)\right]} \geq \frac{\pi}{4 R_{S} \sin \left[\pi \delta_{S}(w) /\left(2 R_{S}\right)\right]}=\sigma(w ; S) .
$$

Thus,

$$
\begin{aligned}
d(0, z ; T)=\int_{\Gamma} \sigma(w ; T)|d w| & \geq \int_{\Gamma} \sigma(w ; S)|d w| \\
& \geq \inf _{C} \int_{C} \sigma(w ; S)|d w|=d(0, z ; S) .
\end{aligned}
$$

(The infimum is over all curves $C$ in the strip $S$ which join 0 and $z$.) Since $\log \operatorname{coth}$ is a decreasing function, $G_{T}(0, z) \leq G_{S}(0, z)$. In addition, if equality holds for some $z$, we also have equality in Minda's result for almost all $w$ on $\Gamma$ and hence $D$ is an infinite strip.

This completes the proof of Lemma 1.

Proof of Lemma 2. It is enough to show the result for the strip resulting from the mapping $\psi(w)=\log ((1+w) /(1-w))$ sending the unit disk onto the strip $\{z=x+i y:-\pi / 2<y<\pi / 2\}$. All other strips with 0 as the center are multiples and rotations of this one. 
The conformal invariance of the Green's function gives that:

$$
\begin{aligned}
& G_{S}(0, z)=-\frac{1}{\pi} \log \left|\psi^{-1}(z)\right|=\frac{1}{\pi} \log \left|\frac{e^{z}+1}{e^{z}-1}\right| \\
& =\frac{1}{2 \pi} \log \left|\frac{e^{2 \rho \cos \theta}+2 e^{\rho \cos \theta} \cos (\rho \sin \theta)+1}{e^{2 \rho \cos \theta}-2 e^{\rho \cos \theta} \cos (\rho \sin \theta)+1}\right| .
\end{aligned}
$$

Differentiating the function inside the logarithm, we see that in order to show that it is decreasing on $[0, \pi / 2)$, it suffices to show:

$$
e^{\rho \cos \theta} \sin (\theta-\rho \sin \theta)-e^{-\rho \cos \theta} \sin (\theta+\rho \sin \theta) \leq 0 .
$$

This involves quite a bit of algebra but can be verified by the reader by hand or with Mathematica.

Proving the inequality above is the same as showing the following:

$$
\begin{gathered}
\frac{e^{\rho \cos \theta}(\rho \cos \theta-1)+e^{-\rho \cos \theta}(\rho \cos \theta+1)}{\rho} \sin (\rho \sin \theta) \\
+\frac{e^{\rho \cos \theta}-e^{-\rho \cos \theta}}{\rho}(\sin (\rho \sin \theta)-\rho \sin \theta \cos (\rho \sin \theta)) \geq 0 .
\end{gathered}
$$

But this holds since in this strip, for $0<\theta<\pi / 2, x=\rho \cos \theta \in(0, \rho), y=$ $\rho \sin \theta \in(0, \pi / 2), e^{x}(x-1)+e^{-x}(x+1) \geq 0$, and $\sin y-y \cos y \geq 0$. This completes the proof of Lemma 2 .

Proof of Corollary 2. It is well known that the following are equivalent ([4], p. 218): (a) $\int_{-\pi}^{\pi} \Phi(g(t)) d t \leq \int_{-\pi}^{\pi} \Phi(h(t)) d t$ for all nondecreasing convex functions on $-\infty<$ $s<\infty$,

(b) $g^{*}(\theta) \leq h^{*}(\theta), 0 \leq \theta \leq \pi$,

(c) $\int_{-\pi}^{\pi}[g(t)-s]^{+} d t \leq \int_{-\pi}^{\pi}[h(t)-s]^{+} d t$ for all real $s$.

Following the proof of Baernstein's theorem in [4], Section 7.3, p. 219-221, we see that, for $0<r<1$ and $\rho>0$,

$$
\int_{-\pi}^{\pi}\left[\log \left|f\left(r e^{i \theta}\right)\right|-\log \rho\right]^{+}=\int_{-\pi}^{\pi}\left[G_{T}\left(0, \rho e^{i \theta}\right)+\log r\right]^{+} d \theta .
$$

If we take $s=\log \rho$, Corollary 2 follows from this, Theorem 1 and the equivalence of $(\mathrm{a})-(\mathrm{c})$.

Remarks. In Nowak [7] and Girela [5], integral mean inequalities are proved for functions which are in BMOA, the space of analytic functions in the unit disk where the boundary values are of bounded mean oscillation on the circle. As explained in [3], the Nowak-Girela results give the following:

Theorem B ([7], [5]). Let $f$ be a univalent function in the unit disk $U$ whose image $f(U)$ has $R_{f(U)}=1$. Suppose $f(0)=0$. Then there is a universal constant $A$ such that for all nonnegative, nondecreasing $\varphi$ on $[0, \infty)$ and all $0<r<1$

$$
\int_{0}^{2 \pi} \varphi\left(\left|f\left(r e^{i \theta}\right)\right|\right) d \theta \leq \int_{0}^{2 \pi} \varphi\left(A\left|\psi\left(r e^{i \theta}\right)\right|\right) d \theta
$$

where $\psi$ is the mapping from $U$ onto the strip of inradius 1 with center at 0 and $\psi(0)=0$. 
Notice that our results in Corollary 2 sharpen this result in the case where $f$ is convex with $f(0)$ the center of the largest disk in $f(U)$. In this case, we can take $A=1$.

The problem of whether one can remove the assumption that $f(0)$ is the center of the largest disk is open. Indeed, Theorem 1 will fail since when one tries to compare $G_{S}(w, z)$ to $G_{S}(0, z)$, a circle $|z|=\rho$ may have more angle inside $S$ when centered at $w$ which is not the center of the strip than when it is centered at 0 which is the center. Thus, for some $\rho$ 's, the Green's function for the strip with pole at $w$ is non-zero on a set of $\theta$ 's of greater measure than the Green's function with pole at 0 . There will be no symmetric decreasing rearrangement result here.

However, integral mean results, such as stated in Corollary 1, still seem to hold. One step in such a proof might be to use geometric arguments to make pointwise comparisons between Green's functions in triangles and strips when the pole is in comparable places inside each domain. However, our geometric arguments relied heavily on the pole being at the center of the domain and do not seem to generalize to poles elsewhere.

Integral mean results such as these are usually proved using the Baernstein star functions, the harmonicity of the extremal function and subharmonicity of the functions to be dominated. However, here as in [3], these techniques seem to fail. Even modified versions of the star-function, adapted to this situation where our extremal function is an odd mapping, fail due to the lack of the subharmonicity of the functions we wish to dominate.

\section{REFERENCES}

1. C. Bandle, Isoperimetric Inequalities and Applications, Pitman, London, 1980. MR 81e:35095

2. R. Bañuelos and T. Carroll, "Brownian Motion and the Fundamental Frequency of a Drum", Duke Math. J. 75 (1994) 575-602. MR 96m:31003

3. R. Bañuelos and E. Housworth, "An Isoperimetric-Type Inequality for Integrals of Green's Functions", Michigan Math. J. 42 (1995) 603-611. MR 96j:30038

4. P. Duren, Univalent Functions, Springer-Verlag, New York, 1983. MR 85j:30034

5. D. Girela, "Inequalities for the Integral Means of BMOA Functions", Complex Variables Theory Appl. 25 (1994) 43-48. MR 95m:30046

6. C. D. Minda, "Lower Bounds for the Hyperbolic Metric in Convex Regions", Rocky Mountain J. Math 13 (1983) 61-69. MR 84j:30039

7. M. Nowak, "Some Inequalities for BMOA Functions, " Complex Variables Theory Appl. 16 (1991) 81-86. MR 92e:30019

8. Ch. Pommerenke, Boundary Behavior of Conformal Maps, Springer-Verlag, New York, 1992. MR 95b:30008

9. R. Sperb, Maximum Principles and Their Applications, Academic Press, New York, 1981. MR 84a:35033

Department of Mathematics, Purdue University, West Lafayette, Indiana 47907

E-mail address: banuelos@math.purdue.edu

Department of Mathematics, University College, Cork, Ireland

E-mail address: tc@ucc.ie

Department of Mathematics, University of Oregon, Eugene, Oregon 97403

E-mail address: eah@math.uoregon.edu 